\title{
Retrospective study of recession of four horizontal rectus muscle in periodic alternating nystagmus
}

This article was published in the following Dove Press journal:

Clinical Ophthalmology

II December 2014

Number of times this article has been viewed

\author{
Osamu Mimura' \\ Hiroto Ishikawa ${ }^{1,2}$ \\ Naoki Kimura' \\ Akiko Kimura' \\ Cesar V Borlongan ${ }^{2}$ \\ 'Department of Ophthalmology, \\ Hyogo College of Medicine, \\ Nishinomiya, Hyogo, Japan; \\ ${ }^{2}$ Department of Neurosurgery and \\ Brain Repair, University of South \\ Florida, Tampa, FL, USA
}

Purpose: Periodic alternating nystagmus (PAN) is a spontaneous horizontal nystagmus observed in disorders of the central nervous system. Patients with congenital PAN complain of oscillating vision at high rates. Medication is the first-choice treatment for PAN; however, clinicians still seek better therapy. The aim of this study was to evaluate outcomes of recession of four horizontal rectus muscle (R-FHR) in patients with congenital PAN.

Patients and methods: This study reports a retrospective case series of ten patients (seven males and three females; mean age 24.4 \pm 10.9 ) with congenital PAN who underwent R-FHR between 2007 and 2012, which was performed by the same surgeon at the Hyogo College of Medicine. Patients were evaluated for complications, recession amount, deviation angle, eye movements including a nystagmus amplitude, and visual acuity during pre- and post-operative periods.

Results: Pre-operatively, patients complained of oscillating vision, abnormal head posture, esotropia, and congenital superior oblique palsy. Post-operatively, changes from the previous observations of nystagmus amplitudes and abnormal head posture demonstrated a complete reversal in all patients. In addition, visual acuity determined with a Snellen chart improved in two patients. However, esotropia occurred in three patients who underwent additional strabismus surgery 2 days after R-FHR. R-FHR was particularly effective in eight patients who preoperatively had periodic oscillating vision with a regular pattern of periodic nystagmus.

Conclusion: We demonstrated that ten patients with congenital PAN had improved vision following R-FHR, indicating that R-FHR was an effective procedure, especially in patients suffering PAN with periodic oscillating vision.

Keywords: congenital nystagmus, null point nystagmus, strabismus surgery, ocular torticollis, oscillating vision, electro-oculogram

\section{Introduction}

Periodic alternating nystagmus (PAN) is a spontaneous horizontal nystagmus observed in disorders of the central nervous system, such as a cerebellar disorder, and is characterized by a fast phase beating in one direction for a period of approximately 2-3 minutes at the primary position. ${ }^{1}$ Recently, the ratio of PAN prevalence was estimated to be between $9 \%{ }^{2}$ and $39 \%{ }^{1}$ in congenital nystagmus. Although PAN can occur during infancy or later in life, patients with congenital PAN complain of oscillating vision because of their null points in comparison to patients with other congenital nystagmus. ${ }^{3,4}$ In Western countries, the first-choice treatment for PAN is medication-based, such as use of baclofen ${ }^{5}$ and memantine. ${ }^{6}$ However, in Japan, the first choice is prismatic glass and the second is surgery, because medication for PAN is not covered by health insurance. Furthermore, previous studies reported that these pharmaceutical interventions have limited effects in congenital PAN.,8 Therefore, we examined the efficacy 
of strabismus surgery; ie, recession of four horizontal rectus muscle (R-FHR).

R-FHR has demonstrated levels of efficacy as a good surgical procedure for patients with null point nystagmus. ${ }^{9,10}$ It is controversial whether R-FHR is effective for treating $\mathrm{PAN}^{9}$ or not, ${ }^{4}$ and currently, there are no reports showing R-FHR efficacy in patients with PAN. Here, we show ten patients who received R-FHR for oscillating vision caused by congenital PAN and demonstrate that the procedure is effective, especially in treating some variants of PAN; the patients complained of oscillating vision mainly.

\section{Materials and methods}

The medical records of ten patients (seven males and three females; $24.4 \pm 10.9$ years) with congenital PAN who underwent R-FHR between November 2007 and July 2012 at the Hyogo College of Medicine were analyzed retrospectively during a follow-up period of at least 6 months (average period 27.8 months). Patients with congenital PAN were extracted for the present study; however, patients with other types of congenital nystagmus including albinism, fusion maldevelopment nystagmus, nystagmus blockade syndrome, convergence dampened nystagmus, and other congenital nystagmus were excluded. The clinical status for each patient is shown in Table 1. All patients underwent ophthalmologic examinations including best-corrected visual acuity using a Snellen chart (then converted into logarithm of the minimum angle of resolution [ $\log M A R]$ ), major amblyoscope for deviation angle measurements, face photographs for abnormal head posture, Titmus stereo test for stereopsis, and electro-oculograms (EOGs) at the pre- and post-operative periods. EOGs were performed to record for nystagmus amplitude and direction; time constant was 3.0 seconds for original waveforms and 0.01 seconds for the speed waveforms. Firstly, the horizontal saccades (distance $40^{\circ}$ ) were recorded for the calibration. Then, the eye movement in the primary position was recorded at $2 \mathrm{~mm} / \mathrm{second}$ of the paper feed speed. We recorded EOGs until at least three alternations of the nystagmus direction were detected or more than 8 minutes.

The therapeutic effects of R-FHR were determined by both subjective complaints, eg, whether the patient could drive without difficulty and/or read the paper without a loupe, and objective complaints such as changes of the nystagmus amplitudes and the abnormal head posture. Investigations were performed according to the Declaration of Helsinki guidelines. Subjects were fully informed about the nature of the procedures and gave their written consent at the beginning of the treatments.

All surgical procedures were performed under general anesthesia by a single surgeon (OM). All patients received R-FHR, equal recession for both the medial and lateral rectus muscles (MR and LR, respectively). Firstly, the maximum amount of MR was removed ( $9 \mathrm{~mm}$ [14.5 mm from limbus] to $11 \mathrm{~mm}$ [ $16.5 \mathrm{~mm}$ from limbus]), and then the same amount of LR was removed from the muscle attachment site $(16 \mathrm{~mm}$ to $18 \mathrm{~mm}$ from limbus).

The study protocol and data collection methods were approved by the Institutional Review Board of the Hyogo College of Medicine. Written informed consent was obtained from all patients including consent for any accompanying images, including the picture of case number 1 (Figure 1). Case number 2 and 9 patients were under the age of 18 years and we therefore obtained permission from their parent(s) for the surgical consent.

\section{Results}

1) Pre-operative clinical signs: nine patients complained of an abnormal head posture (nine face turn, one head tilt, and two chin elevations [Figure 1]; some features overlapped [Table 1]). The face turn fluctuated over time to various degrees in these patients and the orientation reversed in case number 1 (Figure 1) and case number 10. Aside from the face turn cases, there were two esotropia (ET) and one congenital superior oblique palsy cases (ie, case number 6). Also, the other strabismus or eye movement disorder including dissociated vertical deviation and inferior oblique overaction was not observed in these ten cases.

2) Recession amounts: the recession amounts for 40 muscles in all ten patients were as follows: $9 \mathrm{~mm}$ in two patients $(20.0 \%), 10 \mathrm{~mm}$ in seven patients $(70.0 \%)$ and $11 \mathrm{~mm}$ in one patient $(10.0 \%$; Table 1$)$.

3) Post-operative course: all ten patients showed a reversal in severity and fluctuation ranges in both nystagmus and abnormal head posture on the day after the surgery; the nystagmus improved markedly in four patients (case numbers $1,4,9$, and 10), and the abnormal head posture disappeared completely in three patients (case numbers 2,5 , and 6 ). To enhance our manuscript, we uploaded the video file of nystagmus changes before (Supplementary video S1) and after surgery (Supplementary video $\mathrm{S} 2$ ) in case number 1. EOGs showed a decrease of the nystagmus amplitude with periodic alternation in all cases. Figure 2 shows the EOG results for case number 10 , showing a marked reduction of amplitudes after R-FHR. In two patients (case numbers 5 and 7), we 


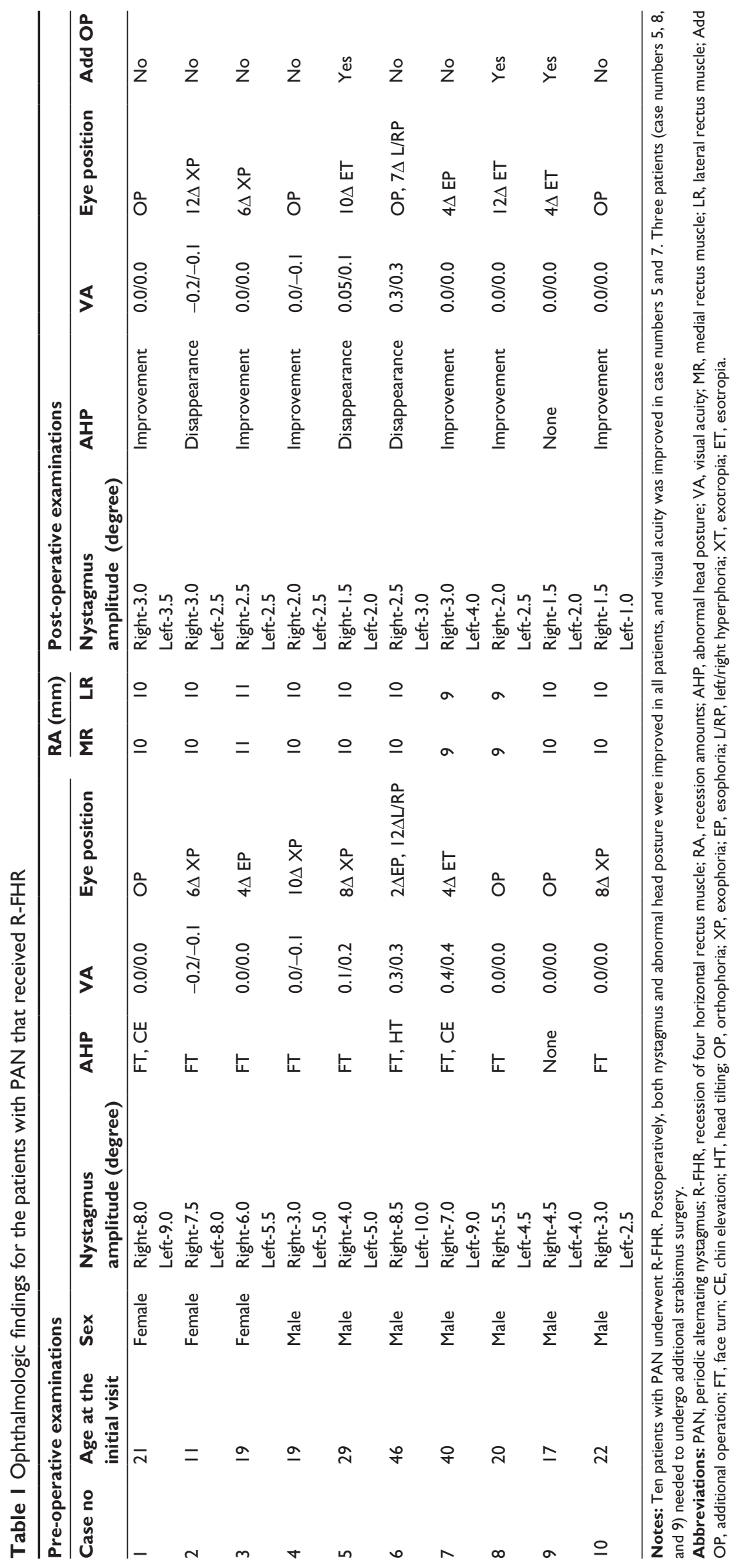




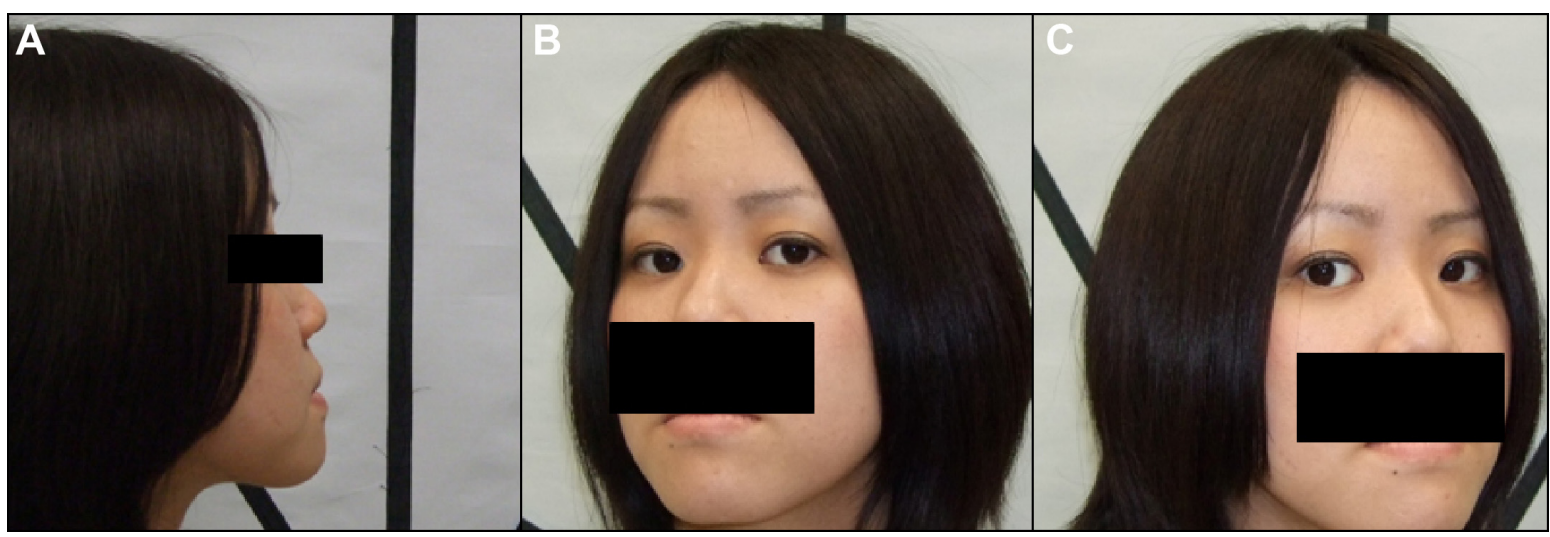

Figure I Abnormal head posture of case number I.

Notes: (A) Shows chin-elevation, (B) and (C) show changes of head posture. Right (B) and left (C) face turns were repeated in alternate shifts for 90 seconds.

observed recovered visual acuity. Furthermore, the recovery in case 7 was 0.4 ( $\log$ MAR; Table 1$)$.

4) Post-operative complications: three patients (case numbers 5, 8, and 9) complained of ET with double vision. They needed to undergo an additional strabismus surgery for hemi advancement of the LR, which led to improvement of the double vision; ie, 2-4 mm LR advancement for ET.

\section{Discussion}

Treatments for correcting nystagmus in Western countries rely mainly on medicine-based interventions. ${ }^{11}$ However, in Japan the main avenue for correcting nystagmus is a prism glass due to the limitations of primary eye care and health insurance. Unfortunately, the effects of a prism glass are limited for correcting PAN. Thus we conducted a research study on R-FHR as the procedure for PAN. Briefly, the rationale for this surgical procedure to correct nystagmus leads to separation of patients into two groups based on correction of abnormal head posture; ie, Anderson- ${ }^{12}$ Kestenbaum- ${ }^{13}$ Goto- ${ }^{14}$ and Parks-method ${ }^{15}$ and reduction of nystagmus amplitude via R-FHR. The majority of patients with PAN have an abnormal head posture such as a face turn. However, the abnormal head posture has a temporal variation; therefore, the surgery for correcting abnormal head posture is an unapproved procedure in patients with PAN. In 1999, Dell'Osso et al first reported the effectiveness of tenotomy and re-attachment of all horizontal rectus muscles for congenital nystagmus, ${ }^{16}$ and the tenotomy resulted in significant
A RE horizontal POS
LE horizontal POS
BE horizontal POS
Pre-operative

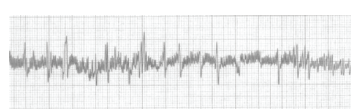

B Post-operative

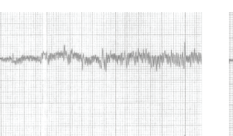

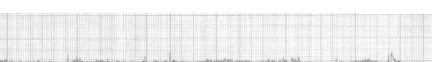

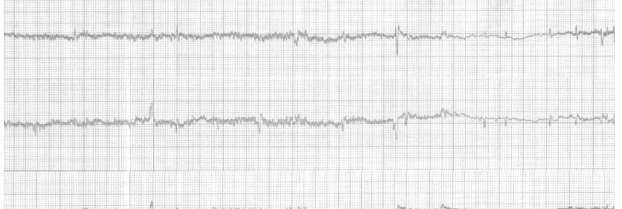

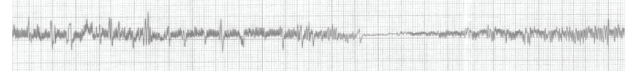

BE horizontal VEL

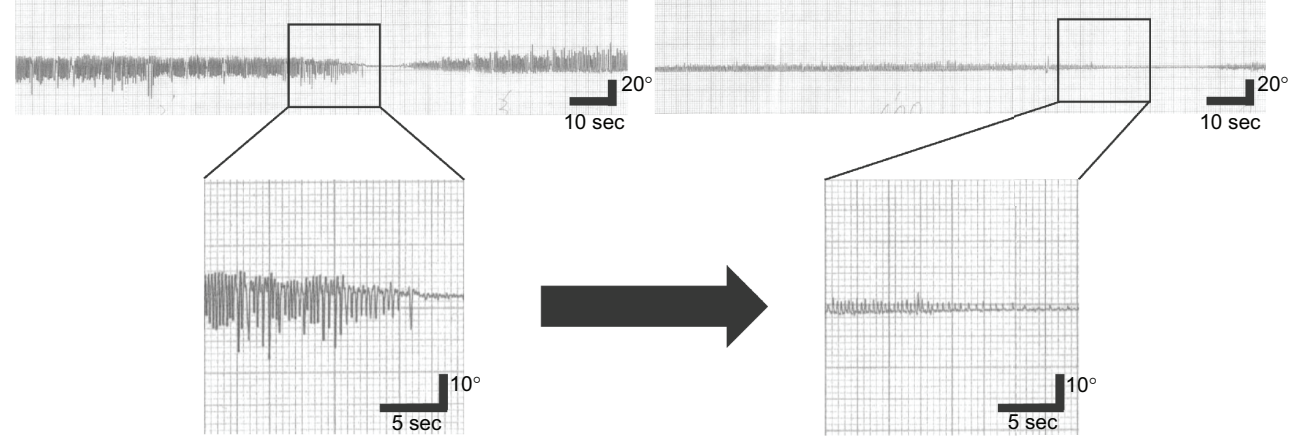

Figure 2 Periodic alternating nystagmus in case number 10 before $(\mathbf{A})$ and after $(\mathbf{B})$ the R-FHR procedure.

Notes: Before R-FHR, PAN reversed direction approximately every 75 seconds and the interlude time was almost 10 seconds; there was horizontal nystagmus. After R-FHR, the amplitudes of nystagmus were markedly decreased, but the periodicity was not changed. Upward deflections indicate rightward eye movements. Time content of position and velocity are 3.0 and 0.01 seconds, respectively.

Abbreviations: PAN, periodic alternating nystagmus; R-FHR, recession of four horizontal rectus muscle; RE, right eye; LE, left eye; BE, both eyes; POS, position; VEL, velocity; sec, seconds. 
improvements in a nystagmus measure and subjective visual functions for ten patients with congenital nystagmus. ${ }^{17}$ They subsequently introduced modifications. ${ }^{18}$ In the past, we also performed the tenotomy; however, unfortunately, we did not experience effectiveness such as described by the Dell'Osso group. We then performed R-FHR to examine its efficacy. Here, we describe the result of a retrospective study of ten patients with congenital PAN who received R-FHR.

R-FHR was first reported by Bietti ${ }^{19}$ in 1956 , but went largely unnoticed until von Noorden and Sprunger; and Helveston et al revaluated R-FHR as a surgical procedure for null point nystagmus. ${ }^{9,10}$ von Noorden and Sprunger performed R-FHR on three patients with congenital nystagmus, including one patient with PAN and abnormal head posture that was corrected, but without vision improvement. ${ }^{9}$ Subsequently, Gradstein et al reported eight patients with PAN who received various surgical procedures in $1997 .{ }^{2}$ The Gradstein team utilized the Kestenbaum method for three patients and R-FHR for five patients. There were no beneficial effects in the former, but correction of abnormal head posture and vision improvement were observed in the latter. In 1996, Thomas et al performed R-FHR for a patient with congenital PAN after a botulin injection to the LR and concluded that R-FHR was not effective based on the patient's subjective improvement with minimal correction of oscillating vision. ${ }^{4}$ Castillo et al performed R-FHR in a patient with secondary PAN who did not respond to baclofen treatment and reported that the nystagmus was reduced, but the patient complained of postoperative exophoria-tropia.? Boyle et al performed R-FHR in ten patients with congenital idiopathic nystagmus and suggested that the visual improvement was only slight. ${ }^{20}$ However, their reports have a few problems with diagnosis of congenital nystagmus. ${ }^{21}$ These observations have led us to be uncertain about R-FHR effects as a surgical procedure for PAN.

In this study, we demonstrated that eight of ten patients with congenital PAN who underwent R-FHR showed an improvement including nystagmus amplitude which was significantly reduced in four patients, abnormal head posture that completely disappeared in three patients, and recovered visual acuity in two patients. Some of these outcome features overlapped. This is especially true for case number 1 , which involved a patient who had refrained from driving due to the oscillating vision, but after the R-FHR procedure, she found herself routinely driving a car, which was clearly indicative of an improvement in the quality of life. R-FHR could not change essentially the location of the null point. However, R-FHR could decrease the nystagmus amplitude and extend the null point over a large area, resulting in the improvement of the abnormal head posture. The remaining three patients showed improvement after additional strabismus surgery.

Previous postoperative complications have indicated the prevalence of exotropia. ${ }^{7}$ However, in our study, the rate of postoperative ET was $30.0 \%$, which had not been reported previously. When we performed the additional strabismus surgery, the removed rectus muscles were attached at the planned site. Therefore, post-operative ET was not caused by a technical issue. Post-operative ET may only occur in people who possess a small orbital volume such as populations of Asian descent. An important clinical consideration can be observed when more than the typical $8 \mathrm{~mm}$ of rectus muscle recession is performed. Bagheri et al suggested that an additional $1 \mathrm{~mm}$ recession had the effect of correcting 6 prism diopters in MR and 11 prism diopters in LR when the recession amount was over the typical $8 \mathrm{~mm} .^{22}$ This previous report supports our hypothesis that more than $8 \mathrm{~mm}$ rectus muscle recession in Asian descent may induce postoperative ET.

\section{Conclusion}

R-FHR is effective for patients with PAN who complain of periodic oscillating vision with a regular pattern of periodic nystagmus. Although post-operative strabismus may occur, due consideration is warranted for reduction of the recession amount. In addition, the preferable age of receiving surgery is over 10 years of age because of the need for topical anesthesia to perform the additional surgery. As a challenge in the future, we need to increase the sample size to further ascertain the detail of R-FHR that is effective for PAN, and to use NAFX, foveation time, and gaze-dependent visual acuity as valid scientific evidence. ${ }^{21}$

\section{Acknowledgment}

We thank Dr David Eve (University of South Florida) for his critical advice, comments, and English editing of the manuscript.

\section{Disclosure}

The authors report no conflicts of interest in this work. None of the authors received funding for this study.

\section{References}

1. Shallo-Hoffmann J, Faldon M, Tusa RJ. The incidence and waveform characteristics of periodic alternating nystagmus in congenital nystagmus. Invest Ophthalmol Vis Sci. 1999;40(11):2546-2553.

2. Gradstein L, Reinecke RD, Wizov SS, Goldstein HP. Congenital periodic alternating nystagmus. Diagnosis and Management. Ophthalmology. 1997;104(6):918-928. 
3. Shallo-Hoffmann J, Riordan-Eva P. Recognizing periodic alternating nystagmus. Strabismus. 2001;9(4):203-215.

4. Thomas R, Mathai A, Braganza A, Billson F. Periodic alternating nystagmus treated with retrobulbar botulinum toxin and large horizontal muscle recession. Indian J Ophthalmol. 1996;44(3):170-172.

5. Troost BT, Janton F, Weaver R. Periodic alternating oscillopsia: a symptom of alternating nystagmus abolished by baclofen. $\mathrm{J} \mathrm{Clin} \mathrm{Neu-}$ roophthalmol. 1990;10(4):273-277.

6. Kumar A, Thomas S, McLean R, et al. Treatment of acquired periodic alternating nystagmus with memantine: a case report. Clin Neuropharmacol. 2009;32(2):109-110.

7. Castillo IG, Reinecke RD, Sergott RC, Wizov S. Surgical treatment of trauma-induced periodic alternating nystagmus. Ophthalmology. 2004;111(1):180-183.

8. Halmagyi GM, Rudge P, Gresty MA, Leigh RJ, Zee DS. Treatment of periodic alternating nystagmus. Ann Neurol. 1980;8(6):609-611.

9. von Noorden GK, Sprunger DT. Large rectus muscle recessions for the treatment of congenital nystagmus. Arch Ophthalmol. 1991; 109(2):221-224.

10. Helveston EM, Ellis FD, Plager DA. Large recession of the horizontal recti for treatment of nystagmus. Ophthalmology. 1991;98(8): 1302-1305.

11. Ehrhardt D, Eggenberger E. Medical treatment of acquired nystagmus. Curr Opin Ophthalmol. 2012;23(6):510-516.

12. Anderson JR. Causes and treatment of congenital eccentric nystagmus. Br J Ophthalmol. 1953;37:267-281.

13. Kestenbaum A. [New operation for nystagmus]. Bull Soc Ophtalmol Fr. 1953;6:599-602.
14. N G. A study of optic nystagmus by the electrooculogram. Acta Soc Ophthalmol Jap. 1954;851-865.

15. Parks MM. Symposium: nystagmus. Congenital nystagmus surgery. Am Orthopt J. 1973;23:35-39

16. Dell'Osso LF, Hertle RW, Williams RW, Jacobs JB. A new surgery for congenital nystagmus: effects of tenotomy on an achiasmatic canine and the role of extraocular proprioception. J AAPOS. 1999;3(3):166-182.

17. Hertle RW, Dell'Osso LF, FitzGibbon EJ, et al. Horizontal rectus tenotomy in patients with congenital nystagmus: results in 10 adults. Ophthalmology. 2003;110(11):2097-2105.

18. Dell'Osso LF, Tomsak RL, Thurtell MJ. Two hypothetical nystagmus procedures: augmented tenotomy and reattachment and augmented tendon suture (Sans tenotomy). J Pediatr Ophthalmol Strabismus. 2009; 46(6):337-344.

19. Bietti GB. [Notes on ophthalmological surgical technics]. Boll Ocul. 1956;35(9-12):642-656. Italian.

20. Boyle NJ, Dawson EL, Lee JP. Benefits of retroequatorial four horizontal muscle recession surgery in congenital idiopathic nystagmus in adults. $J$ AAPOS. 2006;10(5):404-408.

21. Hertle RW, Dell'Osso LF. Benefits of retroequatorial four horizontal muscle recession surgery in congenital idiopathic nystagmus in adults. J AAPOS. 2007;11(3):313-314.

22. Bagheri A, Farahi A, Yazdani S. The effect of bilateral horizontal rectus recession on visual acuity, ocular deviation or head posture in patients with nystagmus. J AAPOS. 2005;9(5):433-437.
Clinical Ophthalmology

\section{Publish your work in this journal}

Clinical Ophthalmology is an international, peer-reviewed journal covering all subspecialties within ophthalmology. Key topics include: Optometry; Visual science; Pharmacology and drug therapy in eye diseases; Basic Sciences; Primary and Secondary eye care; Patient Safety and Quality of Care Improvements. This journal is indexed on

\section{Dovepress}

PubMed Central and CAS, and is the official journal of The Society of Clinical Ophthalmology (SCO). The manuscript management system is completely online and includes a very quick and fair peer-review system, which is all easy to use. Visit http://www.dovepress.com/ testimonials.php to read real quotes from published authors. 